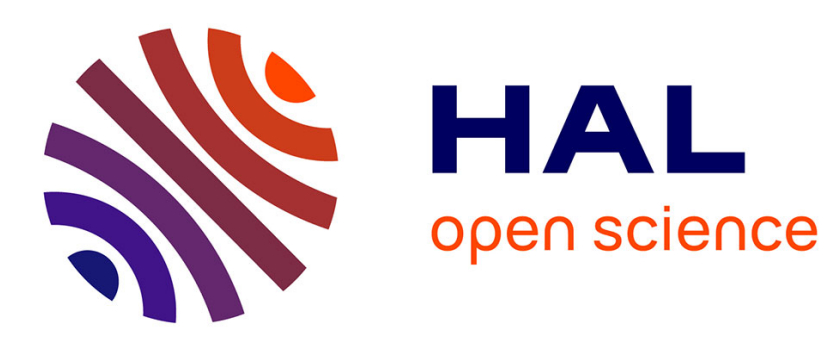

\title{
Improving Efficiency of Data Assimilation Procedure for a Biomechanical Heart Model by Representing Surfaces as Currents
}

\author{
Alexandre Imperiale, Alexandre Routier, Stanley Durrleman, Philippe \\ Moireau
}

\section{To cite this version:}

Alexandre Imperiale, Alexandre Routier, Stanley Durrleman, Philippe Moireau. Improving Efficiency of Data Assimilation Procedure for a Biomechanical Heart Model by Representing Surfaces as Currents. FIMH - 7th International Conference on Functional Imaging and Modeling of the Heart - 2013, Jun 2013, London, United Kingdom. pp.342-351, 10.1007/978-3-642-38899-6_41 . hal-00841442

\author{
HAL Id: hal-00841442 \\ https://hal.inria.fr/hal-00841442
}

Submitted on 4 Jul 2013

HAL is a multi-disciplinary open access archive for the deposit and dissemination of scientific research documents, whether they are published or not. The documents may come from teaching and research institutions in France or abroad, or from public or private research centers.
L'archive ouverte pluridisciplinaire HAL, est destinée au dépôt et à la diffusion de documents scientifiques de niveau recherche, publiés ou non, émanant des établissements d'enseignement et de recherche français ou étrangers, des laboratoires publics ou privés. 


\title{
Improving Efficiency of Data Assimilation Procedure for a Biomechanical Heart Model by Representing Surfaces as Currents
}

\author{
A. Imperiale ${ }^{1}$, A. Routier ${ }^{1,2}$, S. Durrleman ${ }^{2}$ and P. Moireau ${ }^{1}$ \\ 1 Inria Ile-de-France Saclay, M $\exists D I S I M$ Team, Palaiseau, France \\ 2 ICM, Hôpital La Pitié-Salpêtrière, Paris, France
}

\begin{abstract}
We adapt the formalism of currents to compare data surfaces and surfaces of a mechanical model and we use this discrepancy measure to feed a data assimilation procedure. We apply our methodology to perform parameter estimation in a biomechanical model of the heart using synthetic observations of the endo- and epicardium surfaces of an infarcted left ventricle. We compare this formalism with a more classical signed distance operator between surfaces and we numerically show that we have improved the efficiency of our estimation justifying the use of state-of-the-art computational geometry formalism in the data assimilation measurements processing.
\end{abstract}

\section{Introduction}

Data assimilation methods are widely accepted $[1,3,7,8,12,15,16]$ as an essential tool in heart modeling in order to personalize from various sources of measurements - in particular image-data - not only the specific heart geometry of a patient but also the biomechanical parameters describing a particular physiological state - healthy or pathological. A large variety of data assimilation methodologies have already been used in conjunction with various biomechanical models of the heart. This work, however, focuses on the remaining challenge of combining state-of-the-art image segmentation and registration methods - developed initially in static configuration $[4,11,13]$ - with dynamical biomechanical models to extract the maximal amount of information in the data assimilation

procedure. To do so with medical imaging data, we rely on the framework of currents to generate a vector space of surfaces - extracted from segmentations - which proves to be very effective for comparing model and data.

\subsection{Overview of the data assimilation method}

In a general setting we consider a physical system described by a set of timevariables gathered in the so-called system state $x$ following the dynamical model

$$
\dot{x}_{\left[\zeta_{x}, \zeta_{\theta}, \omega\right]}(t)=A(x(t), \theta, \omega(t), t), \quad x_{\left[\zeta_{x}, \zeta_{\theta}, \omega\right]}(0)=x_{0}+\zeta_{x} \quad \text { and } \quad \theta_{\left[\zeta_{\theta}\right]}=\theta_{0}+\zeta_{\theta} \text {, }
$$


where $A$ is a possibly non-linear operator, $\theta$ represents a set of uncertain parameters and $\omega$ an unknown function representing some model error. Moreover, we consider that the initial state $x(0)$ and parameters $\theta$ are composed of known a priori values $x_{0}$ and $\theta_{0}$ whereas $\zeta_{x}$ and $\zeta_{\theta}$ describe potential uncertainties. Then, we assume that the actual system denoted by $x^{\dagger}$ corresponds to a specific instance of this system class which cannot be simulated since we do not know the corresponding actual values $\zeta_{x}^{\dagger}, \zeta_{\theta}^{\dagger}, \omega^{\dagger}$. However, we do have measurements $Z$ on the actual system that we traditionally model by the action of an observation operator $H$ on the state variable, i.e.

$$
Z=H\left(x^{\dagger}\right)+\chi
$$

where $\chi$ denotes a measurement error. In this context, data assimilation aims at using these observations to estimate the actual trajectory $x^{\dagger}$ and also identify the modeling parameters $\theta^{\dagger}$.

Following [9] we choose sequential data assimilation, but since in this work we focus on the definition and use of specific types of observations, most of the results obtained here can be adapted to variational data assimilation methods such as in $[3,12,16]$. In fact, data assimilation procedures are mainly built on the capacity of measuring discrepancy between the estimated trajectory and parameters $(\widehat{x}, \widehat{\theta})$ and the actual trajectory and parameters $\left(x^{\dagger}, \theta^{\dagger}\right)$ through the use of the observation operator. The idea is to reduce a discrepancy measure

$$
E_{D}(Z, \widehat{x})=\frac{1}{2}\|Z-H(\widehat{x})\|_{\mathcal{Z}}^{2},
$$

and rely on the amount of information contained in the observations descriptioni.e. the observability - to ensure the convergence of the estimator to the actual system. Hence, in sequential data assimilation we build a sequential estimator whose dynamic reads

$$
\left\{\begin{array}{l}
\dot{\hat{x}}=A(\widehat{x}, \widehat{\theta}, t)+G_{x}(Z-H(\widehat{x})), \quad \widehat{x}(0)=x_{0}, \\
\dot{\hat{\theta}}=G_{\theta}(Z-H(\widehat{x})), \quad \widehat{\theta}(0)=\theta_{0},
\end{array}\right.
$$

and expect $\left\|\widehat{x}-x^{\dagger}\right\| \rightarrow 0$ and $\left\|\theta-\theta^{\dagger}\right\| \rightarrow 0$ in time as fast as possible. In the expressions (3), $G_{x}$ and $G_{\theta}$ represent both the state and parameter gain operators which are always obtained by analyzing the sensitivity of the discrepancy measure to variations of the state. To fix the idea we summarize the different possibilities by

$$
G_{x}(Z-H(\widehat{x}))=\left(P_{x}+P_{x \theta}\right) \frac{\partial}{\partial x} E_{D}(Z, \widehat{x}), \quad G_{\theta}(Z-H(\widehat{x}))=P_{\theta} \frac{\partial}{\partial x} E_{D}(Z, \widehat{x}),
$$

and various choices are possible for such operators with the Extended Kalman Filtering (EKF) [7], nudging [10] or even the Unscented Kalman Filter (UKF) $[8,15]$ which consists in using particular interpolation point instead of the discrepancy exact tangent operator. In this work, we adopt a hybrid approach made $[1,6]$ of the combination of a simple nudging operator on the large state 
space - namely $P_{x}=\gamma \mathbb{1}$ - which is in charge of reducing the initial state error $\zeta_{x}$ and the model error $\omega$ - that we couple with Reduced-order Unscented Kalman Filtering (RoUKF) in charge of the remaining parameter uncertainty $\zeta_{\theta}$ in oder to define $\left(P_{x \theta}, P_{\theta}\right)$. Therefore in our approach as in all other choices of data assimilation strategies we have to give a definition of the observation operator $H$, the observation space $\mathcal{Z}$ and its norm $\|\cdot\|_{\mathcal{Z}}$.

\subsection{Biomechanical model of the heart}

Before focusing on the data we have to consider a biomechanical model of the heart. We rely on the model extensively described in $[1,12]$ built under the general principle of dynamics in Lagrangian formulation. The state variables are the displacement $y$ of the solid from a reference configuration, its velocity $\underline{v}$ and the internal mechanical variables $e_{c}, k_{c}, \tau_{c}$ describing the strains, active stiffnesses and stresses in the sarcomeres. The state variable becomes $x=\left\{\underline{y}, \underline{v}, e_{c}, \tau_{c}, k_{c}\right\}$. For the sake of simplicity we will assume that the electrical component of this model is provided - by a measurement processing or the use of an electrophysiological model - so that we can focus on the mechanical behavior of the heart. For the same reason the internal pressures in the cavities are prescribed as a function of time, typically obtained from measurements or from another model. On this mechanical heart model, we expect to retrieve some contractility parameters that are relevant to represent infarcted tissues - as already done in other data assimilation investigations such as [1] - in order to illustrate the interest of the introduction of the current formalism for the data processing.

\subsection{Surface based available information}

We assume that the available information is obtained from a prior step of image processing - segmentation of left ventricle endo- and epicardium for instance - and the major difficulty for data assimilation purposes lies in the difference between the Lagrangian formulation of the mechanical model and the Eulerian nature of such data as no model-point to data-point mapping exists in general. One way to circumvent these difficulties - see [1] for Cine-MRI or [6] in the context of tagged-MRI segmentation - is to build a discrepancy measure using the signed distance between the given surface data and the corresponding deformed model geometries. However, this method may suffer from standard disadvantages as illustrated in Figure 1(a) and 1(b). The efficiency of the data assimilation procedure can then be deteriorated in complex geometrical situations like those possibly encountered in the heart. For that reason, we would like to avoid these matching difficulties by considering the formalism of surface currents $[4,14]$ which has been successfully used in the field of medical image analysis and that clearly goes further in terms of data representation than the classical signed distance function. In fact we show (numerically at least) that the obtained observability - which is basically a quantification of how much information on the actual system we manage to extract from the data - is clearly improved. Naturally this improvement has a price in terms of mathematical 
complexity but also in terms of numerical computation as we will see in the sequel.

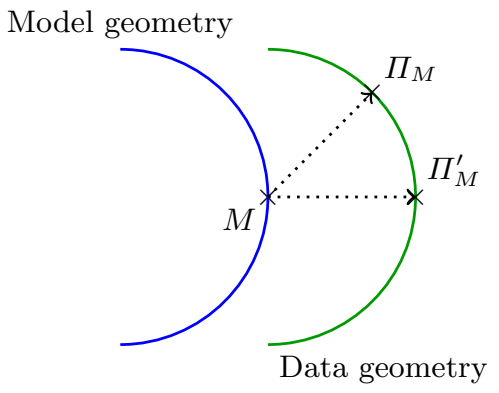

(a) Non uniqueness of the projection point
Data geometry

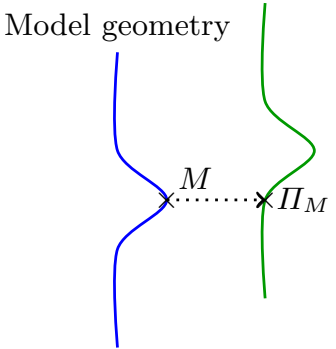

(b) Irrelevant shape discrepancy

Fig. 1. Classical disadvantages of the signed distance operator.

\section{Discrepancy measure using currents formalism}

\subsection{Surfaces as linear forms and R.K.H.S.}

Let $S$ be a surface embedded in $\mathbb{R}^{3}$ with normal vector field $\underline{n}_{S}$. Considering a Hilbert space $\mathcal{W}$ of smooth test vector fields defined within the ambient space, the key idea behind currents is to understand surfaces as operators on such test space. Surfaces are defined by

$$
\forall \underline{w} \in \mathcal{W} \quad\langle S, \underline{w}\rangle_{\mathcal{W}^{\prime} \mathcal{W}}=\int_{S} \underline{w} \cdot \underline{n}_{S} \mathrm{~d} \sigma
$$

which means that the space of surfaces is assimilated with the dual space of $\mathcal{W}$, hence equipped with the classical sup norm. Following $[4,5,17]$ we define $\mathcal{W}$ as a Reproducing Kernel Hilbert Space (RKHS) in order to give a numerically tractable expression of this norm. By definition RKH spaces are Hilbert spaces admitting every evaluation functional in their dual spaces. Using the Riesz representation theorem this feature directly leads to the so-called reproducing property. Namely, there exists a unique two arguments function $K(\cdot, \cdot)$ - called the kernel of $\mathcal{W}$ - such that

$$
\forall \underline{w} \in \mathcal{W}, \forall \underline{x}, \alpha \in \mathbb{R}^{3} \quad \underline{w}(\underline{x}) \cdot \alpha=(K(\cdot, \underline{x}) \alpha, \underline{w})_{\mathcal{W}},
$$

where $(\cdot, \cdot)_{\mathcal{W}}$ stands for the inner product of $\mathcal{W}$. Using the definition (4) and property (5) we can define the unique vector field $\underline{s} \in \mathcal{W}$ that represents the surface $S \in \mathcal{W}^{\prime}$ by

$$
\left.\forall \underline{w} \in \mathcal{W} \quad\langle S, \underline{w}\rangle_{\mathcal{W}^{\prime} \mathcal{W}}=(\underline{s}, \underline{w})_{\mathcal{W}} \quad \text { with } \quad \underline{s}^{\left(x_{1}\right.}\right)=\int_{S} K\left(\underline{x}_{1}, \underline{x}_{2}\right) \underline{n}_{S}\left(\underline{x}_{2}\right) \mathrm{d} \sigma .
$$


Finally the norm of surfaces reads

$$
\|S\|_{\mathcal{W}^{\prime}}^{2}=\left\langle S, \underline{s}_{\mathcal{W}^{\prime} \mathcal{W}}=\iint_{S} \underline{n}_{S}\left(\underline{x}_{1}\right) \cdot K\left(\underline{x}_{1}, \underline{x}_{2}\right) \underline{n}_{S}\left(\underline{x}_{2}\right) \mathrm{d} \sigma \mathrm{d} \sigma\right.
$$

Assuming that the data $Z$ take the form of surfaces, we consider $H(\widehat{y})$ the corresponding deformed model geometry. The data assimilation method will be fed through the following fidelity-to-data term

$$
E_{D}(Z, \underline{\hat{y}})=\frac{1}{2}\|Z-H(\underline{\widehat{y}})\|_{\mathcal{W}^{\prime}}^{2}
$$

Note that in practice the choice of the kernel is crucial since it weights the discrepancy measure. Following [4] we choose a Gaussian kernel, namely $K\left(\underline{x}_{1}, \underline{x}_{2}\right)=$ $\exp \left(-\left|\underline{x}_{1}-\underline{x}_{2}\right|^{2} / \lambda^{2}\right)$. The kernel width $\lambda$ is then calibrated depending on the sizes of the geometric objects at hand but more importantly on the expected level of noise. As a matter of fact, large values of $\lambda$ result in a flatten metric in the space of currents.

\subsection{Derivation of the fidelity-to-data term}

As we explained in Section 1.1, in order to define the dynamics of the observer we need to be able to compute the derivative of the discrepancy measure with respect to the state variable. We denote by $\underline{z}$ and $\underline{h}(\widehat{y})$ the Riesz representation of both data and model geometries and by $\underline{\widehat{\widehat{n}}}$ the estimated normal vector field. Let $\delta y$ be a test displacement, from (4) we can derive the desired expression which after simplications reads

$$
\begin{aligned}
\frac{\partial E_{D}}{\partial \underline{y}} \cdot \underline{\delta y} & =-\left(\frac{\partial H}{\partial \underline{y}}(\underline{\widehat{y}}) \cdot \underline{\delta y}, Z-H(\underline{\widehat{y}})\right)_{\mathcal{W}^{\prime}}=-\left\langle\frac{\partial H}{\partial \underline{y}}(\underline{\widehat{y}}) \cdot \underline{\delta y}, \underline{z}-\underline{h}(\underline{\widehat{y}})\right\rangle_{\mathcal{W}^{\prime} \mathcal{W}} \\
& =-\int_{H} \underline{\underline{\nabla}}(\underline{z}-\underline{h}) \cdot \underline{\delta y} \cdot \underline{\hat{n}} \mathrm{~d} \sigma-\int_{H}(\underline{z}-\underline{h}) \cdot \frac{\partial}{\partial \underline{y}}(\underline{\widehat{n}} \mathrm{~d} \sigma) \cdot \underline{\delta y} .
\end{aligned}
$$

In the first term of (9) we see the spatial derivative of both Riesz representants integrated over the model deformed surface, but even more interesting is the second part where the derivative of the surface element $\underline{\widehat{n}} \mathrm{~d} \sigma$ appears. It is of better interest because it emphasizes a connection between the community of data assimilation, image analysis, computational geometry [17] and shape sensitivity analysis [2] where similar terms are of common use. 


\subsection{Computational aspects}

In practice the model surface is a triangulated surface, therefore integrals in (9) are decomposed over each triangle $\mathcal{T}_{i}$ :

$$
\begin{aligned}
\frac{\partial E_{D}}{\partial \underline{y}} \cdot \underline{\delta y}=\sum_{i=1}^{N_{T}}-\int_{\mathcal{T}_{i}} \underline{\underline{\nabla}}(\underline{z}-\underline{h}) \cdot \underline{\delta y} \cdot \frac{\partial \underline{x}_{i}}{\partial r} \wedge \frac{\partial \underline{\widehat{x}}_{i}}{\partial s} \mathrm{~d} r \mathrm{~d} s \\
\\
\quad-\int_{\mathcal{T}_{i}}(\underline{z}-\underline{h}) \cdot\left(\frac{\partial \delta y}{\partial r} \wedge \frac{\partial \underline{\widehat{x}}_{i}}{\partial s}+\frac{\partial \underline{\widehat{x}}_{i}}{\partial r} \wedge \frac{\partial \delta y}{\partial s}\right) \mathrm{d} r \mathrm{~d} s .
\end{aligned}
$$

In the previous expression $\underline{\underline{x}}_{i}$ is the so-called local map of the $i^{\text {th }}$ triangle of the deformed model surface. Finally, integrals are numerically approximated by evaluating each integrand at the center of the deformed triangle. By doing so we obtain the same terms as in [14] which justifies our adaptation of the currents formalism. Concerning the time discretization, it is important to note that an explicit scheme is prohibited since it leads to inefficient stabilization - or even worse numerical instability - in a data assimilation strategy as demonstrated in [10]. Therefore, as prescribed in [10], we choose a linearized version of an implicit mid-point scheme to obtain

$$
\begin{aligned}
\frac{\partial E_{D}}{\partial \underline{y}}\left(Z, \underline{\hat{y}}^{n+1 / 2}\right) \cdot \underline{\delta y} \approx- & \left(\frac{\partial H}{\partial \underline{y}}\left(\underline{\widehat{y}}^{n+1}\right) \cdot \underline{\delta y}, Z^{n+1 / 2}-H\left(\underline{\widehat{y}}^{n+1}\right)\right)_{\mathcal{W}^{\prime}} \\
& +\left(\frac{\partial H}{\partial \underline{y}}\left(\widehat{\widehat{y}}^{n}\right) \cdot \underline{\delta y}, \frac{\partial H}{\partial \underline{y}}\left(\underline{\widehat{y}}^{n}\right) \cdot\left(\underline{\widehat{y}}^{n+1}-\underline{\widehat{y}}^{n}\right)\right)_{\mathcal{W}^{\prime}} .
\end{aligned}
$$

where $\widehat{y}^{n}$ is the approximation of $\widehat{y}(n \Delta t)$. This leads, however, to an implicit expression with respect to the unknown state variable $\underline{y}^{n+1}$. Let $\underline{v}^{*}, \underline{w}^{*}$ be two admissible displacements. In (10) the following bilinear form appears:

$$
\left(\frac{\partial H}{\partial \underline{y}}\left(\underline{\widehat{y}}^{n}\right) \cdot \underline{v}^{*}, \frac{\partial H}{\partial \underline{y}}\left(\underline{\widehat{y}}^{n}\right) \cdot \underline{w}^{*}\right)_{\mathcal{W}^{\prime}}=\left\langle\frac{\partial H}{\partial \underline{y}}\left(\underline{\widehat{y}}^{n}\right) \cdot \underline{v}^{*}, \frac{\partial \underline{h}}{\partial \underline{y}}\left(\underline{\widehat{y}}^{n}\right) \cdot \underline{w}^{*}\right\rangle_{\mathcal{W}^{\prime} \mathcal{W}} .
$$

Note that after spatial discretization of the space of admissible displacements (typically through a finite element method) and numerical integration of expressions (6) and (9) - as previously detailed - this operator leads to a full matrix of size equals to the number of triangles in the model geometry. Indeed, by contrast with the signed distance operator the observation operator based on currents is non-local, binding every node of the surfaces with each other. Therefore, we can expect better correspondence between surfaces, hence increasing the global observability/identifiability - i.e. the ability to estimate the right trajectory parameters from the measurements. As a counter part it requires some special care in order to keep a reasonable numerical cost when solving the linear systems appearing in the estimation algorithm. Assuming a reasonable number of triangles on the boundary surfaces (from 1000 to 50,000 typically), the bandwidth of the complete finite element matrix stem from (11) is dramatically deteriorated, leading to a significant increase in the computational time. To partially circumvent 
this difficulty we follow a domain decomposition strategy in the final resolution of the problem by iterating between the degrees of freedom of the boundary surfaces and the interior degrees of freedom. As a result, we can use a classical sparse solver for the part of the problem that remains local and use a full matrix solver only on the boundary degrees of freedom. This strategy converges in very few iterations between the interior and the boundary, decreasing significantly the total computational time.

\section{Estimation results using synthetic data}

\subsection{Comparison with signed distances}

To compare the two possible choices of discrepancy operators that we have mentioned we first consider the problem of using the discrepancy measure to stabilize a weakly viscous solid towards the null trajectory from any initial condition. In fact, it was shown in [10] that this type of "toy problem" is relevant for the ability of a data assimilation method to track trajectories. More precisely, we consider a simplified ellipsoidal left ventricle geometry that will represent an elastic material with very low viscosity. The initial condition is the solution of a static elastic problem loaded with a homogeneous pressure inside the cavity. The data consist in the static reference configuration corresponding to null displacement. The direct model is vibrating around this position whereas the filtered dynamics in both cases (i.e. using signed distances or currents) rapidly stabilize as we can see in Figure 2(a) showing the evolution in time of the energy of the computed solution. In these simulations we have chosen the gain parameter $\gamma$ with the help of a spectral analysis of the operator driving the dynamics of the estimation error system [10] . Namely, we choose the gain in order to obtain the smallest spectral abscissa in Figure 2(b) leading to the highest possible stabilization in Figure 2(a). We deduce that the observer using the formalism of currents benefits from a faster stabilization than the one using the signed distance for a large set of eigenpairs, namely possible uncertainty frequencies. Moreover, the spectral analysis allows us to calibrate the kernel width for the best configuration where no noise is present in the data. This ideal value can then be adjusted in real configuration to moderate the data errors.

\subsection{Estimation results and conclusion}

We can now fully illustrate our filtering procedure by performing a complete parameter estimation using synthetic data on a realistic geometry - see Figure 3(a). First, we perform a direct model simulation where we assume the contractility parameter to be significantly lower in a given region (red region in Figure 3(a) where $\theta=0.2$ ) as it could be expected in the presence of infarcted tissue. During this direct simulation we extract endo- and epicardium surfaces of the left ventricle. These data are then used in the estimation simulation starting from the a priori that the whole tissue is healthy ( $\theta=1$ for all regions). In this synthetic 


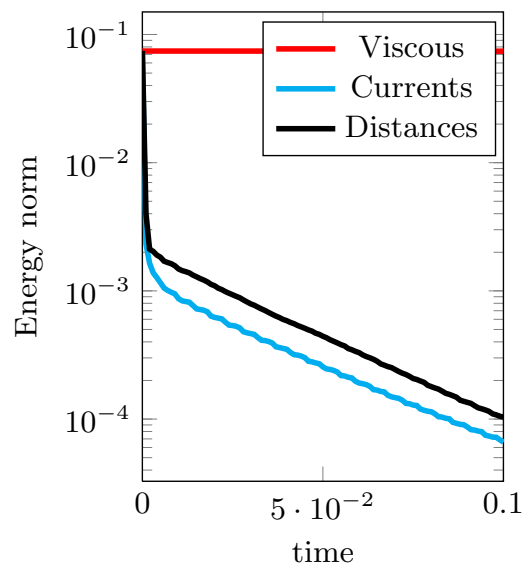

(a) Evolution in time of the energy norm

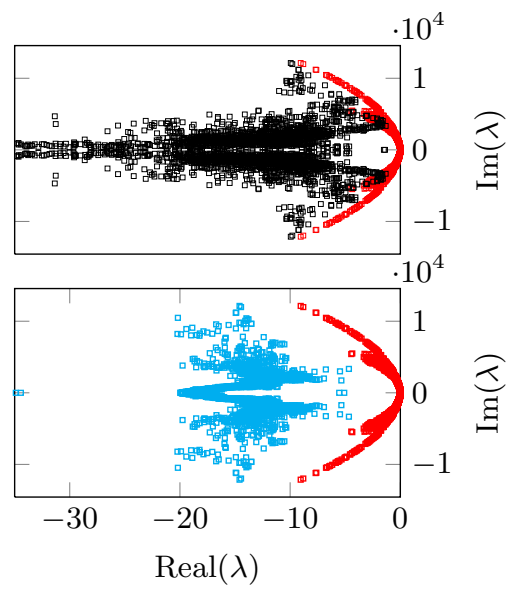

$\square$ Distances $\square$ Currents $\square$ Viscous

(b) Spectral analysis of observers

Fig. 2. Stabilization process using observers calibrated by spectral analysis.

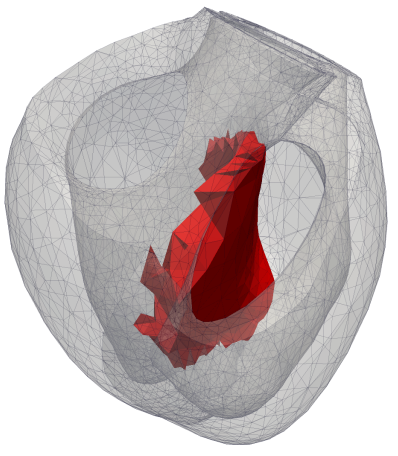

(a) Model view

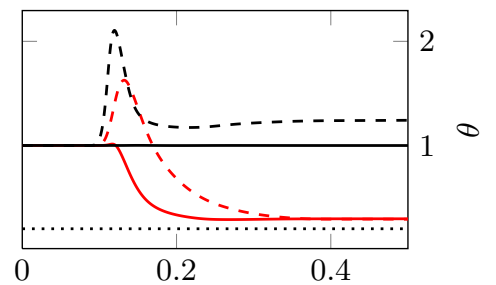

Currents Reg. \#1 - - - Dist. Reg. \#1 Currents Reg. \#2 --- Dist. Reg. \#2

(b) Contractility parameter vs. time

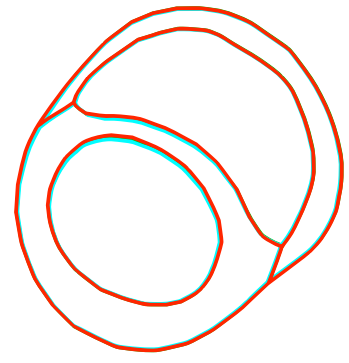

(c) Early systole

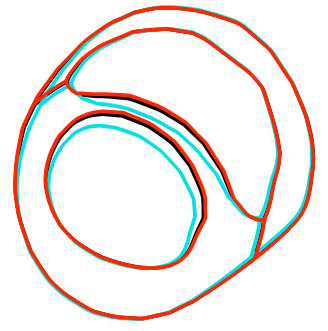

(d) Mid-systole

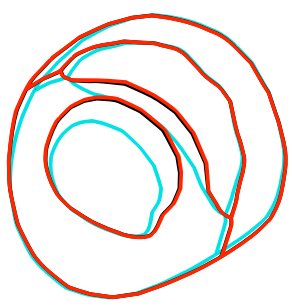

(e) Tele-systole

Fig. 3. Estimation results using synthetic observations with comparison to direct models. Fig. 3(c), 3(d) and 3(e) shows simulations (in short axis view) during heart contraction. Black: estimation, red: infarcted model, cyan: healthy model. 
experiment, the level of expected noise is low since only numerical approximation can affect the definition of the surfaces which we know for sure are the result of an instance of the model. In 3(b) we plot the contractility parameters of the two regions evolving in time during a simulation run, and we see that we retrieve very accurately the ground truth values of the parameters (dotted line in 3(b)) when the heart starts to contract. In terms of resulting displacements for the heart model we see in Figures 3(c), 3(d), 3(e) - in short axis view - that the estimator (in black) has already converged to the actual trajectory (in red) corresponding to the infarcted heart whereas when no identification is performed an assumed healthy heart (in cyan) is far from the measured trajectory. In comparison, the dashed lines in 3(b) show the evolution in time of the estimated parameters obtained using the distance discrepancy operator. Therefore, in the ideal case of synthetic data with a very high confidence in the observations, the global nature of the currents measure shows better performance than the collection of local adjustment from the distance measure.

These preliminary results need further validation by, for instance, increasing the number of estimation regions or by assimilating real data sets where model errors affect the estimation result as described in [1]. However the stabilization benefit showed in Figure 2(a) ensure that the estimation by the current formalism will be robust to a large set of possible errors. On those accounts, the current methodology paves the way of using state of the art fidelity-to-data terms along with full biomechanical models in data assimilation strategies for the biophysical model personalization.

\section{References}

1. R. Chabiniok, P. Moireau, A. Lesault, P.-F.and Rahmouni, J.-F. Deux, and D. Chapelle. Estimation of tissue contractility from cardiac cine-mri using a biomechanical heart model. Biomechanics and modeling in mechanobiology, 2011.

2. M. C. Delfour and J.-P. Zolésio. Shapes and geometries: metrics, analysis, differential calculs, and optimization. Advances in design and control. Society for Industrial and Applied Mathematics, 2011.

3. H. Delingette, F. Billet, K.C.L. Wong, M. Sermesant, K. Rhode, M. Ginks, C.A. Rinaldi, R. Razavi, and N. Ayache. Personalization of Cardiac Motion and Contractility From Images Using Variational Data Assimilation. IEEE Trans. Biomed. Eng., 59(1):20-24, 2012.

4. S. Durrleman, X. Pennec, A. Trouvé, and A. Ayache. Statistical models on sets of curves and surfaces based on currents. Med. Image Anal., 13(5):793-808, Oct. 2009.

5. J. Glaunès, J. Qui, M.I. Miller, and L. Younes. Large deformation diffeomorphic metric curve mapping. Int. J. Comput. Vision, 80(3):317-336, 2008.

6. A. Imperiale, R. Chabiniok, P. Moireau, and D. Chapelle. Constitutive parameter estimation methodology using Tagged-MRI data. FIMH 2011, 2011.

7. H. Liu and P. Shi. Maximum a Posteriori Strategy for the Simultaneous Motion and Material Property Estimation of the Heart. IEEE Trans Biomed Eng., 56(2):378-389, 2009. 
8. S. Marchesseau, H. Delingette, M. Sermesant, and N. Ayache. Fast parameter calibration of a cardiac electromechanical model from medical images based on the unscented transform. Biomecch. Model. Mechan., 2012.

9. P. Moireau and D. Chapelle. Reduced-order unscented kalman filtering with application to parameter identification in large-dimensional systems. Control, Optimisation and Calculus of Variations, 17:380-405, 2011.

10. P. Moireau, D. Chapelle, and P. Le Tallec. Filtering for distributed mechanical dystems using position measurements: perspectives in medical imaging. Inverse Problems, 25, 2009.

11. J. Peters, O. Ecabert, C. Meyer, H. Schramm, R. Kneser, A. Groth, and J. Weese. Automatic whole segmentation in static magnetic resonance image volumes. In Proc. MICCAI 2007, 2007.

12. J. Sainte-Marie, D. Chapelle, R. Cimrman, and M. Sorine. Modeling and estimation of the cardiac electromechanical activity. Comput. Struct., 84:1743-1759, 2006.

13. M. Sermesant, C. Forest, X. Pennec, H. Delingette, and N. Ayache. Deformable biomechanical models: application to $4 \mathrm{D}$ cardiac image analysis. Medical Image Analysis, 7(4):475-88, 2003.

14. M. Vaillant and J. Glaunès. Surface matching via currents. In Proceedings of the 19th international conference on Information Processing in Medical Imaging, volume 3565 of IPMI'05, pages 381-392, Berlin, Heidelberg, 2005. Springer-Verlag.

15. J. Xi, P. Lamata, J. Lee, P. Moireau, D. Chapelle, and N.P. Smith. Myocardial transversely isotropic material parameter estimation from in-silico measurements based on a reduced-order unscented Kalman filter. Journal of the Mechanical Behavior of Biomedical Materials, 4(7):1090-1102, 2011.

16. J. Xi, P. Lamata, S. Niederer, S. Land, W. Shi, X. Zhuang, S. Ourselin, S.G. Duckett, A.K. Shetty, C.A. Rinaldi, D. Rueckert, R. Razavi, and N.P. Smith. The estimation of patient-specific cardiac diastolic functions from clinical measurements. Medical Image Analysis, pages 1-14, 2012.

17. L. Younes. Shapes and diffeomorphisms. Springer, 2010. 